EA 4272

\title{
Health-enhancing activities and the environment: How competition for resources make the environmental policy beneficial
}

\author{
Xavier PAUTREL $\left(^{*}\right)$
}

$2009 / 28$

$\left({ }^{*}\right)$ LEMNA, Université de Nantes

Laboratoire d'Economie et de Management Nantes-Atlantique Université de Nantes

Chemin de la Censive du Tertre - BP 52231

44322 Nantes cedex 3 - France

www.univ-nantes.fr/iemn-iae/recherche

UNIVERSITÉ DE NANTES 


\title{
Health-enhancing activities and the environment: How competition for resources make the environmental policy beneficial
}

\author{
Xavier Pautrel ${ }^{*}$ \\ xavier.pautrel@univ-nantes.fr
}

April 29, 2009

\begin{abstract}
In a two-period overlapping generations model, this paper demonstrates that the relationship between environmental taxation and economic activity (output level and output growth) becomes inverted-U shaped when the detrimental impact of pollution on health and the private decision of each working-age agent to improve her health are taken into account. In particular, a tighter environmental tax is more likely to promote (rather than to harm) output-level and -growth when health is very sensitive to pollution, the weight of health in preferences is high, the polluting capacity of the production technology is high and the rate of natural purification of pollutants is low.

The inverted-U shaped relationship between environmental tax and economic activity is due to a positive effect arising from the competition for resources between the final output sector and the health-enhancing activities. This offsets the conventional detrimental "drag-down effect" for low values of the environmental tax.

We also demonstrate that the link between environmental tax and lifetime welfare is inverted-U shaped as well. Finally, we investigate the social optimum and the determinants of the optimal environmental tax.
\end{abstract}

Keywords : Growth; Environment; Health; Overlapping generations.

*address: Nantes Atlantique Université, Laboratoire d'Économie de Nantes (LEN), Chemin de la Censive du Tertre, BP 52231, 44322 Nantes Cedex 3, France. 


\section{Introduction}

Is environmental policy harmful to economic activity, both in terms of output level and growth? Does the reduction of pollution imply such a heavy cost for the economy that the gains from a better environment quality are not able to offset it? At the theoretical level, the answers are not clear-cut.

The aim of this article is to contribute to the debate focusing on one of the more striking features of pollution: its detrimental impact on health. In contrast to previous works in the field that take into account the impact of pollution on life expectancy (i.e. mortality), we focus our attention on the influence of pollution on illness and disability (morbidity). Indeed, a growing set of empirical evidence finds a link between pollution and chronic diseases such as cancer, diabetes, hypertension, stroke, heart disease, pulmonary conditions and mental disorders, amongst others. Even if pollution is not the main source of these diseases, it contributes as a factor favouring their emergence or their worsening. In contrast to mortality which affects mainly the oldest old, illness and disability due to chronic diseases primarily impacts the working-age population, leading to important losses of productivity and rising health-expenditure for the 30-50 old age people. Devol and Bedroussian (2007), from the Milken Institute, estimate that the seven common chronic diseases represent $\$ 277$ billion spent annually on treatment and a lost productivity equal to $\$ 1.1$ trillion per year for the United-States.

Rising health-expenditure for working-age people and the time they have to devote in order to manage to their chronic disease creates competition for resources that could be used in alternative ways, for example growth-led activities or final production activities. ${ }^{1}$ The main contribution of this paper is to demonstrate that such resource competition is a channel of transmission between the environmental policy, economic activity and welfare, when the detrimental impact of pollution on the health of the working-age population is taken into account.

To demonstrate this point and analyze its implications, we use a two-period overlapping generations model, in the line with previous articles addressing intergenerational environmental issues ${ }^{2}$ introducing an explicit link between the environment and health.

\footnotetext{
${ }^{1}$ Competition for resources in the relation between health and growth has already been studied in several articles (see Dormont et al., 2007, for details and references). Nevertheless, most of these contributions view better health as an increase in life expectancy. Empirically, Dormont et al. (2006) find changes in morbidity that induce savings which more than offset the increase in spending due to population ageing.

${ }^{2}$ For example, John and Pecchenino (1994) who analyze the potential conflict between economic growth and the maintenance of environmental quality when consumption degrades environmental quality while
} 
Following empirical evidence, we assume that health is negatively influenced by pollution but is improved by the investment in health-enhancing activities made by each agent during her working-age. ${ }^{3}$ Pollution is a by-product of final output production and in the competitive economy the government taxes final output to limit pollution emissions.

Our first contribution is to demonstrate that if the detrimental effect of pollution on health-status and an endogenous investment in health by the working-age are taken into account, the link between environmental taxation and economic activity (final output level and growth) is inverted-U shaped. Indeed, a tighter environmental policy has two opposite effects. First, because the environmental tax is imposed on final output, it reduces the rewards to production factor: the conventional "drag-down effect". Second, it reduces pollution and therefore improves health-status of the working-age population. Agents reduce their investment in health-enhancing activities and the freed resources are used to increase consumption and production. This second effect, called the "resource competition effect" is positive. As a result, for low values of the environmental tax, the second effect offsets the first one, and the environmental policy promotes the level and the growth rate of output, as well as overall welfare.

Our second contribution is to show that the greater the room for improvement in the health status, the more likely the environmental policy promotes economic activity and growth. This occurs when the rate of natural health decay is low, the efficiency of health care spending is low, the weight of health in preferences is high, the share of labor in final output is high, the rate of natural purification of pollutants is low, the polluting capacity of production technology is high, and the detrimental impact of pollution on health and the elasticity of pollution stock with respect to the net flow of pollution are high. Most of these criteria exist in the most developed countries, and because the detrimental impact of pollution on health is well-documented worldwide, our results show that an active environmental policy in these countries is highly probable to promote growth and output levels: ie the positive gains in terms of health and growth should be higher than the losses from factor rewards.

Finally, we investigate the social optimum and the optimal environmental tax. We

investment in environmental maintenance promotes it. See also John et al. (1995) who investigate the effects of environmental taxation distinguishing the horizon of the agents and the economy. For models with non-renewable resources, see Agnani et al. (2005), Kemp and Long (1979), Mourmouras (1991, 1993).

${ }^{3}$ Because we consider that agents are suffering from chronic diseases that required medical care when they are young we do not assume that the poor health agents expend more on medical care when they are elderly like Gutiérrez (2008). And, conversely to Williams (2002, 2003), we do not assume that agents who are ill do not work. 
demonstrate that the higher the weight of health in preferences, the elasticity of pollution stock with respect to the net flow of pollution, the detrimental impact of pollution on health and/or the part of labor in production; the higher the optimal environmental tax.

The paper is set out as follows. Section 2 gives empirical evidence on the link between pollution related illness and disability. Section 3 presents the model. Section 4 studies the competitive equilibrium and the impact of environmental taxation on the steady-state. Section 5 investigates the social optimum and the optimal environmental tax. Section 6 examines two extensions: $A K$ endogenous growth and the impact of health on labor productivity. Section 7 concludes.

\section{Pollution, illness and disability}

The majority of environmental economic contributions investigating the detrimental impact of pollution on health focus on life expectancy. ${ }^{4}$ Nevertheless, pollution also affects morbidity by favoring the development of certain chronic diseases that do not always lead to death but have durable detrimental impacts in terms of illness and disability.

These chronic diseases, that include cancer, diabetes, hypertension, stroke, heart disease, asthma and obesity are a growing burden because they represent $60 \%$ of all deaths worldwide and they are a major source of disability (see WHO, 2004, 2005). While they greatly impact developing countries, they also represent a significant burden for developed economies, such as the United-States, England, Canada, Israel, and Australia, as reported by Suhrcke et al. (2006b) and Zhang et al. (2008) among others. Devol and Bedroussian (2007) find that more than half of all Americans (55.8\%) suffered from one or more chronic diseases in 2003.

The causes of increased risk of developing chronic diseases are well-established: mainly unhealthy diet, physical inactivity and tobacco use. Nevertheless, recent studies emphasize the importance of environmental factors in the development and the worsening of some of these chronic diseases, especially in developed countries. According to Briggs (2003) about $8-9 \%$ of the total disease burden may be attributed to pollution in developed countries. ${ }^{5}$

In the case of air pollution for example, it is well-documented that particulate matter pollution, nitrogen dioxide, sulfur dioxide and ozone favour the onset of asthma crises and aggravate respiratory diseases, and that carbon monoxide affects mental function... (for a

\footnotetext{
${ }^{4}$ Among others, see Jouvet et al. (2007), Mariani et al. (2008), Varvarigos (2008) or Pautrel (2008, 2009). The aforementioned articles by Gutiérrez (2008) and Williams (2002, 2003) are exceptions.

${ }^{5}$ See the 2003 special issue of the British Medical Bulletin (issue 1, volume 68) for related references.
} 
study on European countries see Katsouyanni, 2003). Particulate matter air pollution also plays a role in the development (pathogenesis in medical terms) of cardiovascular disease (Brook et al., 2004) and lung cancer (Pope et al., 2002), and it may be particularly harmful to people with diabetes and people with hypertension (Pope and al., 2004). Moreover, air pollution could be deleterious to vascular health especially for the people with diabetes (Rajagopalan et al., 2005; O'Neill et al., 2005). Water pollution [Paulu et al. (1999), Valent et al. (2004)] and industrial pollution [Nadal et al. (2004), Chen and Liao (2005), Schuhmacher and Domingo (2006)] are also reported as detrimental to health.

Lang et al. (2008) recently found that higher unary BPA concentration (chemical pollution) is associated with cardiovascular diagnoses and with diabetes for adults. Smink et al. (2008) show that children in the higher exposure group of HCB (a pesticide extensively used before being banned in the United-States) have an increased risk of being overweight and obese, even if the mother is normal weight. The epidemiological association between persistent organic pollutants and diabetes has been also demonstrated by Rylander et al. (2005) and Porta (2006). Even if such an association does not necessarily prove a causal relation, as noted by Jones et al. (2008), such a relation could be biologically explained (see Remillard and Bunce, 2002).

Furthermore, the link between obesity and diabetes seems to be dependant on pollution. Lee et al. (2007) find that the expected association between obesity and diabetes is absent in people with low concentrations of persistent organic pollutants in their blood. Furthermore, they find that the association between obesity and diabetes become stronger as the concentrations of such pollutants in the blood increase. Lockwood (2002) give further references on the association between exposure to dioxins and the development of diabetes or altered insulin metabolism. He notes that obesity by itself may increase the risk of diabetes: because insulin is concentrated in body fat, obese individuals are likely to have an increased dioxin body burden that could explain the link between the rise in the prevalence of diabetes and the "epidemic" of obesity (see also Ando et al., 2002, for empirical evidence).

One important feature of chronic diseases is that they do not only affect the really old. They also impact working-age people through illness and disability: according to the World Health Organization (WHO, 2004) 56\% of those suffering from some form of disease are people aged 15-59 in high-income countries. Lakdawalla et al. (2004) demonstrate that 
disability increases more rapidly among the young while it decreases for the elderly. They find that one factor is the growth in asthma which appears to be enough to explain the change in disability rate. This is confirmed by Bhattacharya et al. (2008): "Recent work has shown that rates of severe disability, measured by the inability to perform basic activities of daily living, have been rising in working age populations. At the same time, the prevalence of important chronic diseases has been rising, while others falling, among working age populations. Chronically ill individuals are more likely than others to have activity of daily living limitations." Perlkowski and Berger (2004) study the influence of health on working conditions (wages and hours worked) making a distinction between short-term and long-term illness and the age at which each illness appears (because it implies different adjustments for young people at the beginning of careers and for old people closed to retirement). They distinguish between temporary and permanent illness and find that the adverse effects of permanent health problems peak at an age of onset in the 40s for men and in the 30s for women. The biggest decline in wages and hours worked are observed for individuals whose problems started at those ages.

This leads to a major economic impact in terms of labor productivity, labor supply, education or savings, as shown by Suhrcke et al. (2006a). Chronic diseases mainly lead to a reduction of the productivity of the labor-force (even if agents are not sick enough to stop working or even if they are not sick but it is the member of the family who is sick) and the increase in disability of working people. For the US in 2003, Davis et al. (2005) estimated that 55 million workers out of 148 million, aged 19 to 64 reported an inability to concentrate at work due to their own illness or that of a family member and 69 million workers reported missing days due to illness. Regarding the effects of chronic diseases on workers' productivity, Blanc et al. (2001) demonstrate, with a sample of 125 adults in Northern-California that "Both asthma and rhinitis negatively affect work productivity. Those with asthma are less likely to be employed at all, while among those remaining on the job, rhinitis is a more potent cause of decreased work effectiveness. The economic impact of asthma and rhinitis and related conditions may be under-appreciated". For Australia, van Leeuwen et al. (2006) estimate that "while the impact of reduced work effectiveness on days worked with pain on productivity is uncertain, it has the potential to account for the majority of lost productivity costs associated with chronic pain.". Devol and Bedroussian (2007) from the Milken Institute estimate that the seven common chronic diseases represent for the United-States, lost productivity equal to $\$ 1.1$ trillion per year. 
The second important feature of chronic illness is that it places a burden on health care and welfare systems. For the United States in 2003, Devol and Bedroussian (2007) estimate that $\$ 277$ billion is spent annually on treatment. All these resources could be used in alternative activities promoting final (non health) consumption and growth. ${ }^{6}$

Furthermore, chronic illness has major implications in terms of occupational choices, that can not be supported (or funded) by the public health-care system or insurance contracts. Since Grossman's seminal work of 1972, in addition to goods, time appears as an important input in the health production function by influencing the next period's health capital level. More recently, time and time costs in health production have become important in the economic analysis of obesity, for example (see Cutler et al., 2003; Philipson and Posner, 2008, among others). As emphasized by Mullahy and Robert (2008), increasing levels of physical activity is now viewed as a means to improve health outcomes. In their study based on the Bureau of Labor Satistics' American Time Use Survey, Russell et al. (2007) noted that $11.3 \%$ of American adults (in 2003-2004) reported spending time (mean, 108 minutes) on activities related to health on their designed day and 5.6\% (86 minutes) reported making medicine, giving themselves a shot, exercising or therapy for medical reasons. Physical activity has been shown to reduce the risk of developing or dying from heart diseases, diabetes, colon cancer and high blood-pressure. The US Department of Health and Human services advises to be in good health, to do 150 minutes of physical activity at moderate level or 75 minutes at vigourous level each week.

As a result, chronic illness forces agents to allocate more time to health-enhancing activities, time they could use to home or market production. Thus, both the increase in health-care expenditure and the rising investment in health-enhancing activities lead to a competition for resources that could be detrimental for economic activity, growth and/or welfare. And because pollution favours chronic illness and disability, this influence could be a new channel of transmission between the environmental public policy and the economy.

We investigate this point in the following sections.

\footnotetext{
${ }^{6}$ Here, we are reasoning for a given life expectancy and we do not consider that life expectancy may rise or decrease. As a result, we do not integrate the fact that additional years of life increases health-care expenditure (see Suhrcke et al., 2008, p.15).
} 


\section{The model}

Let's consider an overlapping generations model. A new generation is born at each date $t=1,2, \ldots$, and lives for two periods. The number of individuals born at time $t$ is $L$. Population is constant. Individuals are non-altruistic: the old do not care for the young and the young do not care for the old. The preferences of an agent born in period $t$ are represented by the following utility (from van Zon and Muysken (2001)):

$$
\log \left(c_{1 t}^{\phi} h_{t}^{1-\phi}\right)+\theta \log \left(c_{2 t+1}^{\phi} h_{t+1}^{1-\phi}\right)
$$

where $c_{1 t}$ and $c_{2 t+1}$ are respectively consumption in young and old age, $h_{t}$ and $h_{t+1}$ are respectively private health-status in young and in old age. Parameter $\theta=(1+\iota)^{-1}$ where $\iota>0$ is the subjective discount rate of the agent. Parameter $\phi>0$ (respectively $1-\phi$ ) captures the relative importance of consumption (respectively health), in utility. ${ }^{7}$

Each young agent is endowed with one unit of time. She supplies $\nu_{t} \in[0,1[$ of this unit of time in final production and uses her remaining time $1-\nu_{t}$ as an investment in health care activities to improve her health status. ${ }^{8}$ She earns a wage income $\nu_{t} w_{t}$ where $w_{t}$ is the wage rate.

The private health status of an agent born at period $t$ evolves from period $t$ and period $t+1$ according to two opposing forces (Aisa and Pueyo (2004)). On the one hand, biological processes involve a natural decay in health simply as time passes. Following Grossman (1972) and Cropper (1981) we further assume that health depreciates over time with the stock of pollution (denoted $S_{t}$ ). On the other hand, the time invested in health-enhancing activities $\left(1-\nu_{t}\right)$ mitigates against this deterioration. Therefore, for an agent born at $t$, private health status evolves from period $t$ to period $t+1$ as:

$$
h_{t+1}-h_{t}=\eta\left(1-\nu_{t}\right)-\xi S_{t}^{\gamma} h_{t}
$$

with $\eta>0$ is a productivity scalar of health-enhancing activities. ${ }^{9}$. Parameter $\gamma \geq 0$

\footnotetext{
${ }^{7}$ We do not integrate green preferences because we will assume in the following that health status is affected by pollution.

${ }^{8}$ We could assume that there exists a sector that produces health care services with labor and therefore a part $\nu$ of labor is allocated to manufacturing production and a part $1-\nu$ is allocated to health-care production. We would find the same qualitative results (see Appendix A). Consequently, what we call investment in health-enhancing activities could be viewed as health-care expenditure. Our modelling has the advantage of leading to a simpler exposition of the model and the results. In Appendix E, we demonstrate that results are not modified when leisure time is introduced.

${ }^{9}$ Note that here, we model a linear relationship between the health-enhancing activities and the evolution of health-status which could be not empirically relevant. As demonstrated by Skinner et al. (2001): "nearly 20 percent of total Medicare expenditure appears to provide no benefit in terms of survival, nor is it likely that this extra spending improves the quality of life". Our assumption is made for simplicity.
} 
measures the influence of pollution stock on the natural decay $\xi \in] 0,1\left[.{ }^{10}\right.$

A consumer, born at $t$, works during the first period of her life, consumes an amount $c_{1 t}$ and saves the remainder of her revenue. The budget constraint of a young agent is

$$
c_{1 t}+s_{t}=\nu_{t} w_{t}
$$

where $s_{t}$ denotes saving in youth. The budget constraint of an old person is

$$
c_{2 t+1}=\left(1+r_{t+1}\right) s_{t}
$$

where $r_{t+1}$ is the interes rate paid on saving held from period $t$ to $t+1$.

Firms operate through perfect competition using physical capital and labor to produce a final good with a constant return Cobb-Douglas technology:

$$
\left.Y_{t}=\tilde{A}_{t} K_{t}^{\alpha} N_{t}^{1-\alpha}, \quad \alpha \in\right] 0,1[
$$

where $Y_{t}$ is the aggregate output, $K_{t}$ is the aggregate productive capital and $N_{t}$ is labor. $\tilde{A}_{t}$ is a productive scalar, assumed as constant for the moment: $\tilde{A}_{t} \equiv A$. Capital depreciates fully in the production process. ${ }^{11}$

The stock of pollution $S$ from period $t$ to period $t+1$ increases because of the net flow pollution in the current period $t$ and decreases according to the rate of natural purification of pollutants $\mu \in] 0,1[$. The net flow of pollution in period $t$ is the ratio between pollution emissions in period $t$, denoted $E_{t}$, and the abatement activities funded by the government, denoted $D_{t}$. We assume, as conventional, that polluting emissions arise from final production such that

$$
\left.E_{t}=z Y_{t}, \quad z \in\right] 0,1[
$$

Parameter $z$ measures the polluting capacity of the technology. Consequently the stock of pollution in period $t+1$ is defined as:

$$
S_{t+1}=\left(\frac{z Y_{t}}{D_{t}}\right)^{\chi}+(1-\mu) S_{t}
$$

where $\chi>0$ is the exogenous elasticity of pollution stock with respect to the net flow of pollution $E / D$.

\footnotetext{
${ }^{10}$ We impose $\gamma \geq 0$ to investigate the absence of a detrimental impact of pollution on health, that is $\gamma=0$. Nevertheless, it is expected that $\gamma>1$, that is the higher the stock of pollution, the higher the detrimental effect of pollution, even if there is no empirical evidence on such a linear relationship.

${ }^{11}$ The production process is over the course of a generation. If the annual depreciation rate is $10 \%$ (which is empirically relevant), $96 \%$ of the capital stock is depreciated over the course of a 30 year generation. Therefore, we assume that capital is fully used up in the production process. Considering a positive depreciation rate would not change the qualitative results.
} 


\section{Competitive equilibrium}

A representative agent born in period $t$ maximizes her utility function taking wages, the interest rate and the stock of pollution as given. She chooses consumption at both ages $\left(c_{1 t}, c_{2 t+1}\right)$ and the proportion of time $\nu_{t}$ she uses in production:

$$
\begin{array}{r}
\max _{\left\{c_{1 t}, c_{2 t+1}, \nu_{t}\right\}} \log \left(c_{1 t}^{\phi} h_{t}^{1-\phi}\right)+\theta \log \left(c_{2 t+1}^{\phi} h_{t+1}^{1-\phi}\right) \\
\text { s.t. }\left\{\begin{array}{l}
c_{1 t}+s_{t}=\nu_{t} w_{t} \\
c_{2 t+1}=\left(1+r_{t+1}\right) s_{t} \\
h_{t+1}=\eta\left(1-\nu_{t}\right)+\left(1-\xi S_{t}^{\gamma}\right) h_{t}
\end{array}\right.
\end{array}
$$

The first-order condition gives saving:

$$
s_{t}=\left(\frac{\theta}{1+\theta}\right) \nu_{t} w_{t}
$$

and the allocation of time into production:

$$
\left.\nu_{t}=\frac{\phi(1+\theta)}{\eta(1-\phi) \theta} h_{t+1} \quad \in\right] 0,1[
$$

Because $\nu_{t}<1$, the health status of the old $h_{t+1}$ is bounded to $\frac{\eta(1-\phi) \theta}{\phi(1+\theta)} .{ }^{12}$

We assume that abatement $D_{t}$ is provided by the government as a public good and financed by an environmental tax $\tau$ on the source of pollution $Y_{t}$ such that the public budget is balanced at each date: $D_{t}=\tau Y_{t}$. The law of motion of the stock of pollution, given by equation (2) becomes:

$$
S_{t+1}=\left(\frac{z}{\tau}\right)^{\chi}+(1-\mu) S_{t}
$$

Firms maximize their profit $\pi_{t}=(1-\tau) Y_{t}-\left(1+r_{t}\right) K_{t}-w_{t} N_{t}$ and the demand for capital and labor is

$$
\begin{aligned}
& (1-\tau) \alpha Y_{t} / K_{t}=1+r_{t} \\
& (1-\tau)(1-\alpha) Y_{t} / N_{t}=w_{t}
\end{aligned}
$$

The good market clearing yields:

$$
K_{t+1}=s_{t} L
$$

and the labor market clearing equates labor demand $N_{t}$ to labor supply $\nu_{t} L$

$$
N_{t}=\nu_{t} L
$$

\footnotetext{
${ }^{12}$ See van Zon and Muysken $(1997$, p.5) for a justification of the health status boundary. .
} 
The competitive equilibrium may be summarized by the following relations:

$$
\begin{aligned}
& K_{t+1}=(1-\alpha)\left(\frac{\theta}{1+\theta}\right)(1-\tau) A K_{t}^{\alpha}\left(\nu_{t} L\right)^{1-\alpha} \\
& \nu_{t}=\frac{\phi(1+\theta)}{\eta(1-\phi) \theta} h_{t+1} \Rightarrow \nu_{t}=\left(\frac{(1-\phi) \theta}{\phi(1+\theta)}+1\right)^{-1}\left(1+\left(1-\xi S_{t}^{\gamma}\right) \frac{h_{t}}{\eta}\right) \\
& h_{t+1}=\eta\left(1-\nu_{t}\right)+\left(1-\xi S_{t}^{\gamma}\right) h_{t} \\
& S_{t+1}=\left(\frac{z}{\tau}\right)^{\chi}+(1-\mu) S_{t}
\end{aligned}
$$

The steady-state is defined here as an equilibrium where physical capital, private health-status, pollution stock and the allocation of labor in production are constant at $K^{\star}, h^{\star}, S^{\star}$ and $\nu^{\star}$ respectively, defined as:

$$
\begin{aligned}
S^{\star} & =\mathcal{S}(\tau) \equiv \frac{(z / \tau)^{\chi}}{\mu} \\
h^{\star} & =\mathcal{H}(\tau) \equiv \eta\left[\frac{\phi(1+\theta)}{(1-\phi) \theta}+\xi\left(\frac{(z / \tau)^{\chi}}{\mu}\right)^{\gamma}\right]^{-1} \\
\nu^{\star} & =\mathcal{V}(\tau) \equiv \phi\left[\phi+\frac{\xi(1-\phi) \theta}{(1+\theta)}\left(\frac{(z / \tau)^{\chi}}{\mu}\right)^{\gamma}\right]^{-1}
\end{aligned}
$$

Consequently, the health status and the allocation of time in production are positively affected by the environmental $\operatorname{tax} \tau$.

From equations (5), (10) and (11), the steady-state value of the physical capital stock is:

$$
K^{\star}=\mathcal{A}(1-\tau)^{\frac{1}{1-\alpha}} \mathcal{V}(\tau)
$$

with $\mathcal{A} \equiv\left((1-\alpha)\left(\frac{\theta}{1+\theta}\right) A\right)^{1 /(1-\alpha)} L$. Because $Y^{\star}=\frac{1+\theta}{\theta(1-\alpha)}(1-\tau)^{-1} K^{\star},{ }^{13}$ we obtain the steady-state value of final output as a function of the environmental taxation $\tau:$

$$
Y^{\star}=\mathcal{A}_{1}(1-\tau)^{\frac{\alpha}{1-\alpha}}\left(\mathcal{B}+\xi\left(\frac{z^{\chi}}{\mu}\right)^{\gamma} \tau^{-\chi \gamma}\right)^{-1}
$$

with $\mathcal{A}_{1} \equiv \mathcal{B}\left(\frac{1+\theta}{\theta(1-\alpha)}\right) \mathcal{A}$ and $\mathcal{B} \equiv \frac{\phi(1+\theta)}{(1-\phi) \theta}$.

\footnotetext{
${ }^{13}$ Note that $K_{t+1}=s_{t} L=\left(\frac{\theta}{1+\theta}\right) \nu_{t}(1-\tau)(1-\alpha) Y_{t} / \nu_{t}=\left(\frac{\theta}{1+\theta}\right)(1-\tau)(1-\alpha) Y_{t}$.
} 
Proposition 1. When endogenous investment in private health-status and the detrimental impact of pollution on health are taken into account, the relationship between the steady-state output and environmental taxation has an inverted-U shape.

Below (respectively above) an environmental tax-level denoted $\hat{\tau}$ and defined as

$$
\chi \gamma \xi\left(\frac{z^{\chi}}{\mu}\right)^{\gamma} \hat{\tau}^{-1}-\frac{\alpha}{1-\alpha} \mathcal{B} \hat{\tau}^{\chi \gamma}-\left(\frac{\alpha}{1-\alpha}+\chi \gamma\right) \xi\left(\frac{z^{\chi}}{\mu}\right)^{\gamma}=0 .
$$

a tighter environmental taxation raises (respectively lowers) the steady-state level of output $Y^{\star}$.

Proof. See Appendix B

To understand the basic mechanism of this result, let us remember that

$$
Y^{\star}=\mathcal{A}_{1} \underbrace{(1-\tau)^{\frac{\alpha}{1-\alpha}}}_{I a} \underbrace{\mathcal{V}(\tau)}_{I b}
$$

Environmental tax influences the steady-state level of output through two channels: the direct impact of the environmental taxation on the rewards to labor (see overbrace Ia in equation 13) and the (indirect) impact on the allocation of labor in the manufacturing sector (see overbrace $I b$ in equation 13 ).

The former (negative) is the conventional "drag-down" effect of the environmental tax that reduces factor rewards - captured by $(1-\tau)^{\frac{\alpha}{1-\alpha}}$. The latter (positive) is a new channel of transmission due to the "competition for resources" between health enhancing activities and production activities that affects the supply of labor into the final production sector $\nu^{\star}=\mathcal{V}(\tau)$. Because pollution has a detrimental impact on the evolution of private healthstatus, by reducing the net flow of pollution and therefore the stock of pollution, the environmental policy improves the private health-status of the agents. Consequently, each agent decides to reduce her investment in health enhancing activities $\left(1-\nu^{\star}\right.$ decreases $)$ and to raise her labor supply to productive activities ( $\nu^{\star}$ increases). In this way, the tighter environmental tax frees ressources that were allocated to health enhancing activities and that are now reallocated to production, leading to a higher level of steady-state output and a steady-state physical capital. Consequently the competition for resources between output production and health enhancing activities associated with the negative impact of pollution to health is a source of a new channel trough which the environmental policy may promote economic activity. 
When $\gamma=0$, the evolution of health-status is independent from pollution and therefore the investment of each agent in health-enhancing activities is not affected by the environmental tax: $\nu^{\star}$ is independent from $\tau$. In such a case, the competition for resources is not affected by the environmental policy and only the "drag-down"effect remains: the environmental policy is detrimental for growth. In the same way, the "competition for resources effect" no longer holds when there is no endogenous investment in health-enhancing activities.

Proposition 2. Endogenous investment in health and the detrimental impact of pollution on health are two necessary conditions to obtain Proposition 1.

Proof. See above and Appendix B.

Considering the influences of parameters on the environmental tax-level $\hat{\tau}$, enables us to understand why these two opposite effects of the environmental policy leads to an inversed-U shaped relationship between the environmental tax and the steady-state output level. These influences are summarized in the following table (see equation (12) in Proposition 1 and Appendix B for the demonstration):

\begin{tabular}{ccccccc}
\hline$\xi$ & $\eta$ & $\phi$ & $\theta$ & $\alpha$ & $\mu$ & $z$ \\
\hline- & - & - & + & - & - & + \\
\hline
\end{tabular}

Table 1. Parameter Changes and Responses of $\hat{\tau}$

Because it is cumbersome to obtain analytically the influence of $\gamma$ and $\chi$ on the tax-level $\hat{\tau}$, we use a numerical application. We first calibrate the model assuming that the length of each period is 30 years, as usual in the literature. The first period covers ages 20 to 50 , and the second period covers ages 50 to 80 . We use the U.S. economy as a benchmark. From De La Croix and Michel (2002), we choose $\alpha$ and $\theta$ following the standard choice in the RBC literature, that is $\alpha=0.36$ and a quarterly psychological discount factor equal to 0.99 . This implies that $\theta=0.99^{(4 \times 30)}=0.3$. We use the calibration by van Zon and Muysken (1997) for the values of $\xi$ and $\phi$. Finally, parameter $\eta$ is chosen to obtain a private health-status higher than unity to enable the welfare to be positive.

Benchmark value of parameters are summarized in Table 2:

\begin{tabular}{cccccccccc}
\hline$\alpha$ & $\theta$ & $\phi$ & $\xi$ & $A$ & $\eta$ & $L$ & $\chi$ & $\mu$ & $\gamma$ \\
\hline 0.36 & 0.3 & $1 / 2$ & 0.2 & 50 & 0.8 & 1 & 1 & 0.5 & 1.5 \\
\hline
\end{tabular}

Table 2. Benchmark value of parameters 
and the results of the numerical application is reported in Table 3

\begin{tabular}{l|cccccc}
\hline & $\hat{\tau}$ & $\hat{Y}$ & $\hat{\nu}^{\star}$ & $\hat{h}^{\star}$ & $\hat{S}^{\star}$ & $\hat{W}^{\star}$ \\
\hline \hline Benchmark & $21.6 \%$ & 9.73 & 0.896 & 2.068 & 1.84 & 1.28 \\
\hline \hline$\gamma=0.5$ & $7.77 \%$ & 10.77 & 0.90 & 2.089 & 5.15 & 1.46 \\
$\gamma=1$ & $15.73 \%$ & 10.12 & 0.894 & 2.065 & 2.54 & 1.35 \\
$\gamma=2$ & $25.97 \%$ & 9.48 & 0.901 & 2.080 & 1.54 & 1.23 \\
$\gamma=2.5$ & $29.13 \%$ & 9.31 & 0.907 & 2.094 & 1.37 & 1.20 \\
\hline$\chi=1.25$ & $23.14 \%$ & 9.769 & 0.909 & 2.099 & 1.667 & 1.28 \\
$\chi=1.5$ & $24.07 \%$ & 9.821 & 0.921 & 2.125 & 1.515 & 1.29 \\
$\chi=1.75$ & $24.65 \%$ & 9.875 & 0.930 & 2.146 & 1.387 & 1.293 \\
$\chi=2$ & $25.01 \%$ & 9.928 & 0.937 & 2.163 & 1.279 & 1.298 \\
\hline
\end{tabular}

Table 3. Steady-state $\hat{\tau}$ for different values of $\gamma$ and $\chi$

The third proposition stems from the Table 1 and Table 3 .

Proposition 3. When the negative impact of the environment on health and the endogenous decision of each agent to invest her resources in health enhancing activities are taken into account, we demonstrate that the environmental taxation will be more likely to improve the steady-state level of output if the rate of natural health decay $(\xi)$ is low, the efficiency of health care spending $(\eta)$ is low, the weight of health in preferences $(1-\phi)$ is high, the share of labor in final output $(1-\alpha)$ is high, the rate of natural purification of pollutants $(\mu)$ is low, the polluting capacity of production technology $(z)$ is high, and the detrimental impact of pollution on health $(\gamma)$ and the elasticity of pollution stock with respect to the net flow of pollution $\chi$ are high.

Proof. See Appendix B and Table 3.

When the detrimental effects of a dirty environment on health are great, the gains in terms of health status of reducing the pollutant emissions are very significant and the "competition for resources effect" that leads to an increase in labor supply has a greater influence than the "drag-down effect" that reduces factor rewards and as a consequence saving and physical capital accumulation. Neverthless, these positive gains diminish with the increase in the tax rate because the possible improvements in health-status due to the tax are reduced. At the same time, the losses from the reduction of factor rewards increase in the tax rate such that for the environmental tax-level $\hat{\tau}$, they offset the gains, and a further increase in $\tau$ leads to a decrease in the steady-state output level.

Consequently, the greater the room for improving the environment and private healthstatus through environmental policy, the more beneficial the environmental policy is likely 
to be for the economy.

The numerical application also enables us to investigate the impact of environmental taxation on the steady-state lifetime welfare, as well. As shown by Figure 1, there is a relationship that is inverted-U shaped for similar reasons as the relationship between the steady-state output and the environmental tax is inverted-U shaped.

Figure 1: Steady-state lifetime welfare as a function of $\tau$

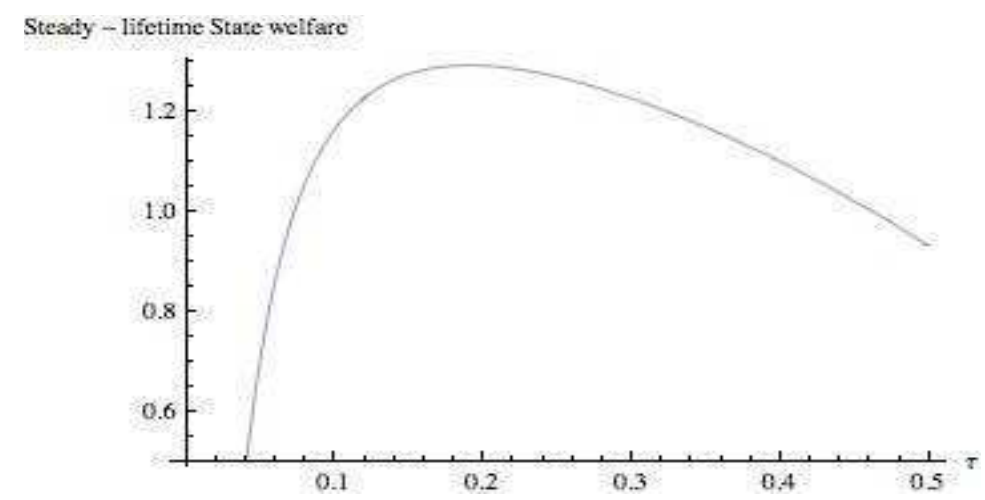

\section{Social optimum and the optimal environmental taxation}

The purpose of this section is to investigate the determinants of the optimal environmental taxation in the presence of endogenous investment in private health and the detrimental impact of pollution on private health.

In the centralized economy, the central planner aims at maximizing the welfare of agents:

$$
\begin{array}{r}
\max _{\left\{c_{1}, c_{2}, \nu, K, D\right\}} \log \left(c_{1}^{\phi} h^{1-\phi}\right)+\theta \log \left(c_{2}^{\phi} h^{1-\phi}\right) \\
\text { s.t. }\left\{\begin{array}{l}
Y=A K^{\alpha}(\nu L)^{1-\alpha}=L c_{1}+L c_{2}+D+K \\
h=\eta(1-\nu) /\left(\xi S^{\gamma}\right) \\
S=(E / D)^{\chi} / \mu \\
E=z Y
\end{array}\right.
\end{array}
$$

As demonstrated in Appendix C, consumption at a young and old age are related ${ }^{14}$

$$
\bar{c}_{1}=\theta \bar{c}_{2}
$$

\footnotetext{
${ }^{14} \mathrm{~A}$ bar ${ }^{-}$denotes optimal value.
} 
with

$$
\bar{c}_{1}=\frac{(1-\alpha) \phi}{\alpha(1+\theta)}\left(\frac{A \alpha \phi}{(1-\alpha) \chi \gamma(1-\phi)+\phi}\right)^{1 /(1-\alpha)}
$$

The optimal allocation of time to production is:

$$
\bar{\nu}=\phi
$$

and the optimal stock of physical capital is

$$
\bar{K}=\left(\frac{A \alpha \phi}{(1-\alpha) \chi \gamma(1-\phi)+\phi}\right)^{1 /(1-\alpha)} \phi L
$$

Consequently, the optimal final output is

$$
\bar{Y}=A^{1 /(1-\alpha)}\left(\frac{\alpha \phi}{(1-\alpha) \chi \gamma(1-\phi)+\phi}\right)^{\alpha /(1-\alpha)} \phi L
$$

Abatement activity is given by

$$
\bar{D}=\frac{(1-\alpha) \chi \gamma(1-\phi)}{(1-\alpha) \chi \gamma(1-\phi)+\phi} \bar{Y}
$$

and the optimal stock of pollution in the steady-state is

$$
\bar{S}=\left(z \frac{(1-\alpha) \chi \gamma(1-\phi)+\phi}{(1-\alpha) \chi \gamma(1-\phi)}\right)^{\chi} / \mu
$$

Consequently, the optimal value of the environmental tax that enables the decentralized economy to attain the optimal stock of pollution in the steady-state is

$$
\bar{\tau}=\frac{(1-\alpha) \chi \gamma(1-\phi)}{(1-\alpha) \chi \gamma(1-\phi)+\phi}
$$

The following proposition arises from this expression.

Proposition 4. The higher the weight of health in preferences $(1-\phi)$, the elasticity of pollution stock with respect to the net flow of pollution $(\chi)$, the detrimental impact of pollution on health (captured by $\gamma$ ) and/or the share of labor in production $(1-\alpha)$; the higher the optimal environmental tax is.

Proof. From equation (14), it is straightforward that $\partial \bar{\tau} / \partial \phi<0, \partial \bar{\tau} / \partial \chi>0, \partial \bar{\tau} / \partial \gamma>0$ and $\partial \bar{\tau} / \partial \alpha<0$.

Nevertheless, an optimal environmental tax is not sufficient to enable the steadystate equilibrium to be optimal because in the decentralized economy the agents do not internalize the impact of their labor supply decisions on final output and the net flow of 
pollution. Consequently, agents do not supply enough time to output production. Hence, to obtain the optimal individual labor supply $\bar{\nu}$, the government have to subsidise healthenhancing activities at a rate (see Appendix D):

$$
\overline{\tau^{\nu}}=1-\frac{\xi \theta}{(1+\theta)}\left(\frac{z^{\chi}}{\mu}\right)^{\gamma}\left(\frac{(1-\alpha) \chi \gamma(1-\phi)}{(1-\alpha) \chi \gamma(1-\phi)+\phi}\right)^{-\gamma \chi}
$$

The environmental tax $\bar{\tau}$ associated with the subsidy $\overline{\tau^{\nu}}$ make the steady-state decentralized equilibrium optimal.

\section{Extensions}

\section{1 $A K$ endogenous growth}

In this section, we consider that external learning by doing à la Romer (1986) exists ${ }^{15}$ such that the productivity scalar $\tilde{A}_{t}$ evolves as physical capital:

$$
\tilde{A}_{t}=A K_{t}^{\alpha}
$$

to obtain an interest rate independent from physical capital.

Consequently, the final output becomes

$$
Y_{t}=A K_{t}\left(\nu_{t} L\right)^{1-\alpha}
$$

and the law of motion of physical capital is given by

$$
K_{t+1}=(1-\alpha)\left(\frac{\theta}{1+\theta}\right)(1-\tau) A K_{t}\left(\nu_{t} L\right)^{1-\alpha}
$$

At the steady-state, physical capital and output evolves at a constant positive rate of growth $g^{\star} \equiv K_{t+1} / K_{t}-1$, that is, using equation (11)

$$
g^{\star}=\mathcal{A}^{\prime}(1-\tau)\left[\mathcal{B}+\xi\left(\frac{z^{\chi}}{\mu}\right)^{\gamma} \tau^{-\chi \gamma}\right]^{\alpha-1}
$$

with $\mathcal{A}^{\prime} \equiv(1-\alpha)\left(\frac{\theta}{1+\theta}\right) A L^{1-\alpha} \mathcal{B}^{1-\alpha}$. The influence of the environmental taxation on the growth rate at the steady-state is given by

$$
\begin{aligned}
\partial g^{\star} / \partial \tau=-\mathcal{A}^{\prime}\left[\mathcal{B}+\xi\left(\frac{(z / \tau)^{\chi}}{\mu}\right)^{\gamma}\right]^{\alpha-1-1} & \tau^{-\chi \gamma} \times \\
& {\left[\mathcal{B}+\xi\left(\frac{z}{\mu}\right)^{\gamma}\left(1+(1-\alpha) \chi \gamma\left(1-\tau^{-1}\right)\right)\right] }
\end{aligned}
$$

\footnotetext{
${ }^{15}$ Following Romer (1986), production factors remain paid at their marginal after environmental tax cost.
} 
Consequently $\partial g^{\star} / \partial \tau>0$ if the last term in the right-hand side of the previous expression is negative:

$$
\mathcal{B} \tau^{\chi \gamma}+\xi\left(\frac{z}{\mu}\right)^{\gamma}\left(1+(1-\alpha) \chi \gamma\left(1-\tau^{-1}\right)\right)<0
$$

Because the left-hand side of the inequality is a monotonic increasing function of $\tau$ with $\lim _{\tau \rightarrow 0}=-\infty$ and $\lim _{\tau \rightarrow 1}=\mathcal{B} \tau^{\chi \gamma}+\xi\left(\frac{z}{\mu}\right)^{\gamma}>0$, there exists a unique $\hat{\tau}_{g}$ defined as

$$
\mathcal{B} \hat{\tau}_{g}^{\chi \gamma}+\xi\left(\frac{z}{\mu}\right)^{\gamma}\left(1+(1-\alpha) \chi \gamma\left(1-\hat{\tau}_{g}^{-1}\right)\right)=0
$$

such that for $\tau<\hat{\tau}_{g}$ (respectively $\tau>\hat{\tau}_{g}$ ) we have $\partial g^{\star} / \partial \tau>0\left(\right.$ resp. $\left.\partial g^{\star} / \partial \tau<0\right)$.

Proposition 5. Under the assumption of a learning-by-doing source of growth à la Romer (1986), the introduction of an endogenous private health care expenditure and a detrimental impact of pollution on health makes the environmental taxation policy good for growth when the level of taxation is not too high.

Proof. See above.

\subsection{Health affects labor productivity}

As emphasized in the introduction, chronic diseases affect the economy through the huge losses of productivity they create. As a result, it is expected that a tighter environmental taxation will reduce this productivity loss by reducing pollution and increasing the health-status of workers. To investigate how the "productivity effect" associated with the "competition for resources effect" could further improve the beneficial impact of an environmental policy, we introduce the impact of health on the productivity of labor.

We continue to consider that poor health agents expend more on medical care when they are young and not elderly as does Gutiérrez (2008). We do not assume that ill agents do not work (Williams $(2002,2003)$ ). Instead we consider that a better health-status makes workers more productive and that absenteeism due to illness does not occur. ${ }^{16}$

The technology to produce final output becomes:

$$
Y_{t}=A_{t} K_{t}^{\alpha}\left(h_{t}^{\varepsilon} N_{t}\right)^{1-\alpha}
$$

\footnotetext{
${ }^{16}$ We take into account presenteeism, i.e. a worker present but with reduced productivity rather than absenteeism, i.e. a worker absent, because it accounts for not only worker health but also health of his family. For the US in 2003, Davis et al. (2005) estimated that 55 million workers over 148 million ages 19 to 64 reported the inability to concentrate at work because of their own illness or that of their family.
} 
where $\varepsilon \geq 0$ measures the effect of health on labor productivity. The introduction of health-dependent labor productivity leaves the model unchanged except for the law of motion of physical capital (equation 5):

$$
K_{t+1}=(1-\alpha)\left(\frac{\theta}{1+\theta}\right)(1-\tau) A K_{t}^{\alpha}\left(h_{t}^{\varepsilon} \nu_{t} L\right)^{1-\alpha}
$$

As a result, the steady-state value of physical capital becomes:

$$
K^{\star \star}=\mathcal{A}(1-\tau)^{\frac{1}{1-\alpha}} \mathcal{V}(\tau) \mathcal{H}(\tau)^{\varepsilon}
$$

and the steady-state expression of final output is now:

$$
Y^{\star \star}=\mathcal{A}_{2}(1-\tau)^{\frac{\alpha}{1-\alpha}}\left(\mathcal{B}+\xi\left(\frac{z^{\chi}}{\mu}\right)^{\gamma} \tau^{-\chi \gamma}\right)^{-(1+\varepsilon)}
$$

with $\mathcal{A}_{2} \equiv \mathcal{A}_{1} \eta^{\varepsilon}$.

Proposition 6. When the effect of health on labor productivity is taken into account, the positive effect of an environmental tax on ouput-level is enhanced and the tax level under which a thigher environmental tax increases output level is higher. The environmental policy is more likely to increase final output.

Proof. The tax level, denoted $\hat{\hat{\tau}}$, for which $\partial Y / \partial \tau=0$ is

$$
(1+\varepsilon) \chi \gamma \xi\left(\frac{z^{\chi}}{\mu}\right)^{\gamma} \hat{\hat{\tau}}^{-1}-\frac{\alpha}{1-\alpha} \mathcal{B} \hat{\hat{\tau}} \chi \gamma-\left(\frac{\alpha}{1-\alpha}+(1+\varepsilon) \chi \gamma\right) \xi\left(\frac{z^{\chi}}{\mu}\right)^{\gamma}=0 .
$$

It is straightforward that for $\varepsilon=0, \hat{\tau}=\hat{\tau}$. Furthermore the LHS of the equation is increasing in $\varepsilon$ because $\hat{\hat{\tau}} \in] 0,1[$. Therefore from the theorem of implicit function we find that $\partial \hat{\hat{\tau}} / \partial \varepsilon>0$. Therefore $\hat{\hat{\tau}}>\hat{\tau}$ when $\varepsilon>0$.

\section{Conclusion}

This paper investigates how environmental tax affects the economy (output-level and growth, welfare) when the detrimental impact of pollution on health is taken into account and working-age individuals have to invest in health-care activities to limit the damaging influence of pollution.

In a two-period overlapping generations model, this paper demonstrates that the relationship between environmental taxation and economic activity ( output level and output growth) becomes inverted-U shaped, when the detrimental impact of pollution on health and the private decision of each working-age agent to improve her health are taken into 
account. In particular, a tighter environmental tax is more likely to promote (rather than to harm) output-level and -growth when health is very sensitive to pollution, the weight of health in preferences is high, the polluting capacity of the production technology is high and the rate of natural purification of pollutants is low.

The inverted-U shaped relationship between environmental tax and economic activity is due to a positive effect arising from the competition for resources between the final output sector and the health-care sector that offsets the conventional detrimental "dragdown effect" for low values of the environmental tax.

We also demonstrate that the link between environmental tax and lifetime welfare is inverted-U shaped as well. Finally, we investigate the social optimum and the determinants of the optimal environmental tax.

This contribution shows that, besides life expectancy, there are other ways in which pollution may affect health and health affect economic activity. Those ways may be new channels of transmission of the environmental pollution to economic activity.

\section{References}

Agnani, B., Amaia, I., and Gutierrez, M. (2005). Growth in overlapping generation economies with non-renewable resources. Journal of Environmental Economics and Management, 50(2):300-318.

Aisa, R. and Pueyo, F. (2004). Endogenous longevity, health and economic growth: a slow growth for a longer life? Economics Bulletin, 9(3):1-10.

Ando, A., Yatagai, T., Rokkaku, K., Nagasaka, S., Ishikawa, S., and Ishibashi, S. (2002). Obesity is a critical risk factor for worsening of glucose tolerance in a family with the mutant insulin receptor. Diabetes Care, 25(8):1484-1485.

Bhattacharya, J., Choudhry, K., and Lakdawalla, D. (2008). Chonic disease and severe disability among working-age populations. Medical Care, 46(1):92-100.

Blanc, P., Trupin, L., Eisner, M., Earnest, G., Katz, P., Israel, L., and Yelin, E. H. (2001). The work impact of asthma and rhinitis: Findings from a population-based survey. Journal of Clinical Epidemiology, 54:610-618.

Briggs, D. (2003). Environmental pollution and the global burden of disease. British Medical Bulletin, 68(1):1-24. 
Brook, R., Franklin, B., Cascio, W., Hong, Y., Howard, G., Lipsett, M., Luepker, R., Mittleman, M., Samet, M., Smith, S., and Tager, I. (2004). Air pollution and cardiovascular disease: a statement for healthcare professionials from the expert panel on population and prevention science of the american heart association. Circulation, 109:26655-2671.

Chen, S. and Liao, C. (2005). Health risk assessment on humans exposed to environmental polycyclic aromatic hydrocarbons pollution sources. Science of the Total Environment.

Cropper, M. (1981). Measuring the benefits from reduced morbidity. The American Economic Review, 71:235-240.

Cutler, D., Glaeser, E., and Shapiro, J. (2003). Why have americans become more obese? Journal of Economic Perspectives, 17(3):93-118.

Davis, K., Collins, S., Doty, M., Ho, A., and Holmgren, A. (2005). Health and productivity amoung u.s. workers. Issue brief, The Commonwealth Fund.

De La Croix, D. and Michel, P. (2002). A Theory of Economic Growth : dynamics and policy in overlapping generations. Cambridge University Press.

Devol, R. and Bedroussian, A. (2007). An unhealthy america: The economic burden of chronic disease - charting a new course to save lives and increase productivity and economic growth. Technical report, Milken Institute.

Dormont, B., Grignon, M., and Huber, H. (2006). Health expenditure growth: Reassessing the threat of ageing. Health Economics, 15(9):947-963.

Dormont, B., Martins, J., Pelgrin, F., and Suhrcke, M. (2007). Health expenditures, longevity and growth. Technical report.

Grossman, M. (1972). On the concept of health capital and the demand for health. Journal of Political Economy, 80:223-255.

Gutiérrez, M. (2008). Dynamic inefficiency in an overlapping generation economy with pollution and health costs. Journal of Public Economic Theory, 10(4):563-594.

John, A. and Pecchenino, R. (1994). An overlapping generations model of growth and the environment. The Economic Journal, 104:1393-1410.

John, A., Pecchenino, R., Schimmelpfenning, D., and Schreft, S. (1995). Short-lived agents and the long-lived environment. Journal of Public Economics, 58:127-141. 
Jones, O., MAguire, M., and Griffin, J. (2008). Environmental pllution and diabetes: a neglected association. Lancet, 371:287-288.

Jouvet, P., Pestieau, P., and Ponthière, G. (2007). Longevity and environmental quality in an olg model. Working paper 2007-19, Université Paris X.

Katsouyanni, K. (2003). Ambient air pollution and health. British Medical Bulletin, 68(1):143-156.

Kemp, M. and Long, N. (1979). The under-exploitation of natural resources: A model with overlapping generations. Economic Record, 55:214-221.

Lakdawalla, D., Bhattacharya, J., and Goldman, D. (2004). Are the young becoming mre disabled. Health Affairs, 23(1):168-176.

Lang, A., Galloway, T., Scarlett, A., Henley, W., Depledge, M., Wallace, R., and Melzer, D. (2008). Association of urinary bisphenol a concentration with medical disorders and laboratory abnormalities in adults. The Journal of the American Medical Association, 300(11):1303-1310.

Lee, D.-H., Lee, I.-K., Jin, S.-H., Steffes, M., and Jocbs, D. (2007). Association between serum concentrations of persistent organic pollutants and insulin resistance among nondiabetic adults: results from the national health and nutrition examination survey. Diabetes Care, 30:622-628.

Lockwood, A. (2002). Diabetes and air pollution. Diabetes Care, 25(8):1487-1488.

Mariani, F., Perez-Barahona, A., and Raffin, N. (2008). Life expectancy and the environment. Technical report, CES Working Paper 2008.48.

Mourmouras, A. (1991). Competitive equilibria and sustainable growth in a life-cycle model with natural resources. Scandinavian Journal of Economics, 93(4):585-591.

Mourmouras, A. (1993). Conservationist government policies and intergenerational equity in an overlapping generations model with renewable resources. Journal of Public Economics, 51(2):249-268.

Mullahy, J. and Robert, S. (2008). No time to lose? time constraints and physical activity. Technical report, NBER Working Paper 14513. 
Nadal, M., Schuhmacher, M., and Domingo, J. (2004). Metal pollution of soils and vegetation in an area with petrochemical industry. Science of the Total Environment, 321:59-69.

O'Neill, M., A.Veves, Zanobetti, A., Sarnat, J., Gold, D., Economides, P., Horton, E., and Schwartz, J. (2005). Diabetes enhances vulnerability to particulate air pollutionassociated impairment in vascular reactivity and endothelial function. Circulation, 111:2913-2920.

Paulu, C., Aschengrau, A., and Ozonoff, D. (1999). Tetrachloroethylene-contaminated drinking water in Massachusetts and the risk of colon-rectum, lung, and other cancers. Environmental Health Perspectives, 107(4):265-271.

Pautrel, X. (2008). Reconsidering the impact of pollution on long-run growth when pollution influences health and agents have a finite-lifetime. Environmental and Resource Economics, 40(1):37-52.

Pautrel, X. (2009). Pollution and life expectancy: How environmental policy can promote growth. Ecological Economics, 68(4):1040-1051.

Perlkowski, J. and Berger, M. (2004). The impact of health on employment, wages, and hours worked over life-cycle. The Quarterly Review of Economics and Finance, 44:102121.

Philipson, T. and Posner, R. (2008). Is the obsety epidemic a public health problem? a decade of research on the economics of obesity. Technical report, NBER Working Paper 14010.

Pope, C. and al. (2004). cardiovascular mortality and long-term exposure to particulate air pollution: epidemiological evidence of general pathological pathways of disease. Circulation, 109(1):71-77.

Pope, C., Burnett, R., Thun, M., Calle, E., Krewski, D., Ito, K., and Thurston, G. (2002). Lung cancer, cardiopulmonary mortality, and long-term exposure to fine particulate air pollution. The Journal of American Medical Association, 287:1132-1141.

Porta, M. (2006). Persistent organic pollutants and te burden of diabetes. Lancet, 368:558559. 
Rajagopalan, S., Sun, Q., and Chen, L. C. (2005). Particulate pollution and endothelial function: Déjà vu all over again in the air. Circulation, 111(2869-2871).

Remillard, R. and Bunce, N. (2002). Linking dioxins to diabetes: epidemiology and biological plausibility. Environmental Health Perspectives, 110:853-858.

Romer, P. (1986). Increasing returns and long run growth. Journal of Political Economy, 94:1002-1037.

Russell, L., Ibuka, Y., and Abraham, K. (2007). Health-related activities in the american time use survey. Medical Care, 45(7):680-685.

Rylander, L., Rignell-Hydbom, A., and Hagmar, L. (2005). A cross-sectional study of the association between persistent organochlorine pollutants and diabetes. Environmental Health, 4:28.

Schuhmacher, M. and Domingo, J. (2006). Long-term study of environmental levels of dioxins and furans in the vicinity of a municipal solid waste incinerator. Environment International, 32:397-404.

Skinner, S., Fisher, E., and Wenberg, J. (2001). The efficiency of medicare. National Bureau of Economic Researh Working paper 8395, National Bureau of Economic Research.

Smink, A., Ribas-Fito, N., Garcia, R., Torrent, M., Mendez, M., Grimalt, J., and Sunyer, J. (2008). Exposure ti hexachlorobenzene during pregnancy increases the risk of overweight in children aged 6 years. Acta Pediatrica, 97:1465-1469.

Suhrcke, M., Arce, R., Mckee, M., and Rocco, L. (2008). Economic costs of ill healthin the european region. Background document, World Health Organization.

Suhrcke, M., Mckee, M., Stuckler, D., Arce, R., Tsolova, S., and Mortensen, J. (2006a). The contribution of health to the economy in the european union. Public Health, 120:994-1001.

Suhrcke, M., Nugent, R., Stuckler, D., and Rocco, L. (2006b). Chronic disease: An economic perspective. Technical report, Oxford Health Alliance, London.

Valent, F., Little, D., Bertollini, R., Nemer, L., Barbone, F., and Tamburlini, G. (2004). Burden of disease attributable to selected environmental factors and injury among children and adolescents in Europe. The Lancet, 363:2032-2039. 
van Leeuwen, M., Blyth, F., March, L., Nicholas, M., and Cousins, M. J. (2006). Chronic pain and reduced work effectiveness: The hidden cost to australian employers. European Journal of Pain, 10:161-166.

van Zon, A. and Muysken, J. (1997). Health, education and endogenous growth. Technical Report 97-009, MERIT Research Memorandum.

van Zon, A. and Muysken, J. (2001). Health and endogenous growth. Journal of Health Economics, 20:169-185.

Varvarigos, D. (2008). Envvironmental quality, life expectancy and sustainable economic growth. Working paper 08/19, University of Leicester, Departement of Economics.

WHO (2004). The global burden of disease: 2004 update. Technical report, World Health Organization.

WHO (2005). Preventing chronic diseases: A vital investment (who, geneva, 2005). Technical report, World Health Organization, Geneva.

Williams, R. (2002). Environmental tax interactions when pollution affects health or productivity. Journal of Environmental Economics and Management, 44(2):261-270.

Williams, R. (2003). Health effects and optimal environmental taxes. Journal of Public Economics, 87:323-335.

Zhang, X., Zhao, X., and Harris, A. (2008). Chronic diseases and labour force participation in australia. Journal of Health Economics.

\section{Appendix}

\section{A The basic model with a health sector}

Let us consider in this section, that there exists in the economy a health sector that produces an amount $H_{t}$ of health-care services at period $t$, using labour and the following technology

$$
H_{t}=A_{H}\left(1-\nu_{t}\right)
$$

where $A_{H}$ is a productivity scalar and $1-\nu_{t}$ is the part of labour (normalized to unity) allocated to the health sector. 
Firms in the health sector operate under perfect competition and maximize their profit $m_{t} H_{t}=w_{t}\left(1-\nu_{t}\right)$ such that:

$$
m_{t} A_{H}=w_{t}
$$

The final output sector is defined as in section 2 .

In the competitive equilibrium, besides her consumption of final good, the consumer buys when she is young $H_{t}$ units of health care service for an amount of health-care expenditure equal to $m_{t} H_{t}$. The private health status of the agent evolves between period $t$ and $t+1$ as:

$$
h_{t+1}-h_{t}=\eta H_{t}-\xi S_{t}^{\gamma} h_{t}
$$

The program of the consumer consists in choosing consumption when young and old and health-care services $H_{t}$ in order to maximize her lifetime utility subject to her budget constraint when young and old and the evolution of her health-status:

$$
\begin{array}{r}
\max _{\left\{c_{1 t}, c_{2 t+1}, H_{t}\right\}} \log \left(c_{1 t}^{\phi} h_{t}^{1-\phi}\right)+\theta \log \left(c_{2 t+1}^{\phi} h_{t+1}^{1-\phi}\right) \\
\text { s.t. }\left\{\begin{array}{l}
c_{1 t}+m_{t} H_{t}+s_{t}=w_{t} \\
c_{2 t+1}=\left(1+r_{t+1}\right) s_{t} \\
h_{t+1}=\eta H_{t}+\left(1-\xi S_{t}^{\gamma}\right) h_{t}
\end{array}\right.
\end{array}
$$

The first-order condition gives saving (using equation 17):

$$
s_{t}=\left(\frac{\theta}{1+\theta}\right)\left(w_{t}-m_{t} H_{t}\right)=\left(\frac{\theta}{1+\theta}\right) \nu_{t} w_{t}
$$

Furthermore, $\nu_{t}$ is given by

$$
h_{t+1}=\frac{\eta A_{H}(1-\phi) \theta}{\phi(1+\theta)} \nu_{t}
$$

that is $\nu_{t}$ increases in $h_{t+1}$. These two expressions are similar to those found when healthcare investment is in the form of time (see equations (3) and (4)) when $A_{H}=1$.

\section{B Environmental taxation in competitive equilibrium}

The influence of environmental tax on the steady-state level of output is given by:

$$
\begin{aligned}
d Y^{\star} / d \tau=Y^{\star} \tau^{-\gamma \chi}(1-\tau)^{-1} & \left(\mathcal{B}+\xi\left(\frac{z^{\chi}}{\mu}\right)^{\gamma} \tau^{-\chi \gamma}\right)^{-1} \times \\
& {\left[\chi \gamma \xi\left(\frac{z^{\chi}}{\mu}\right)^{\gamma} \tau^{-1}-\frac{\alpha}{1-\alpha} \mathcal{B} \tau^{\chi \gamma}-\left(\frac{\alpha}{1-\alpha}+\chi \gamma\right) \xi\left(\frac{z^{\chi}}{\mu}\right)^{\gamma}\right] . }
\end{aligned}
$$


The influence of environmental tax on the steady-state level of output is positive if and only if

$$
\chi \gamma \xi\left(\frac{z^{\chi}}{\mu}\right)^{\gamma} \tau^{-1}-\frac{\alpha}{1-\alpha} \mathcal{B} \tau^{\chi \gamma}-\left(\frac{\alpha}{1-\alpha}+\chi \gamma\right) \xi\left(\frac{z^{\chi}}{\mu}\right)^{\gamma}>0
$$

Because the left-hand side of the inequality is a decreasing monotonic function of $\tau$ with $\lim _{\tau \rightarrow 0}=+\infty$ and $\lim _{\tau \rightarrow 1}=\frac{-\alpha}{1-\alpha}\left(\mathcal{B}+\xi\left(\frac{z^{\chi}}{\mu}\right)^{\gamma}\right)<0$, there exists a unique $\left.\left.\tau \in\right] 0,1\right]$ under which the inequality is verified. This $\tau$ is denoted $\hat{\tau}$ and is defined as:

$$
\chi \gamma \xi\left(\frac{z^{\chi}}{\mu}\right)^{\gamma} \hat{\tau}^{-1}-\frac{\alpha}{1-\alpha} \mathcal{B} \hat{\tau}^{\chi \gamma}-\left(\frac{\alpha}{1-\alpha}+\chi \gamma\right) \xi\left(\frac{z^{\chi}}{\mu}\right)^{\gamma}=0 .
$$

When $\gamma=0$, the left-hand side of the inequality is independent from $\tau$ and negative. Consquently, when $\gamma=0$, we have $d Y^{\star} / d \tau<0$.

To find how parameters affect the tax level $\tau$, let rewrite the expression of $\hat{\tau}$ as:

$$
\Gamma(\hat{\tau} ; \alpha, \gamma, \xi, \mu, \mathcal{B}, z, \chi) \equiv \mathcal{B} \hat{\tau}^{\chi \gamma}+\left(1-\alpha^{-1}(1-\alpha) \chi\left(\hat{\tau}^{-1}-1\right) \gamma\right) \xi\left(\frac{z^{\chi}}{\mu}\right)^{\gamma}=0 .
$$

with $\mathcal{B} \equiv \eta \frac{\phi(1+\theta)}{(1-\phi) \theta}$ and $\hat{\tau}^{-1}>1$. Except for $\gamma$ and $\chi$, it is straightforward that $\partial \Gamma(\cdot) / \partial \xi>$ $0, \partial \Gamma(\cdot) / \partial \mathcal{B}>0, \partial \Gamma(\cdot) / \partial \alpha>0, \partial \Gamma(\cdot) / \partial \mu>0, \partial \Gamma(\cdot) / \partial z<0, \partial \Gamma(\cdot) / \partial \hat{\tau}>0$. From the theorem of implicit function, we obtain

$$
\begin{aligned}
& \partial \hat{\tau} / \partial \xi<0, \quad \partial \hat{\tau} / \partial \eta<0, \quad \partial \hat{\tau} / \partial \phi<0, \quad \partial \hat{\tau} / \partial(1-\phi)>0, \quad \partial \hat{\tau} / \partial \theta>0, \\
& \partial \hat{\tau} / \partial \alpha<0, \quad \partial \hat{\tau} / \partial \mu<0, \quad \partial \hat{\tau} / \partial z>0 .
\end{aligned}
$$

\section{The optimum}

In the centralized economy, the central planner aims at maximizing the welfare of the agents:

$$
\begin{array}{r}
\max _{\left\{c_{1}, c_{2}, \nu, K, D\right\}} \log \left(c_{1}^{\phi} h^{1-\phi}\right)+\theta \log \left(c_{2}^{\phi} h^{1-\phi}\right) \\
\text { s.t. }\left\{\begin{array}{l}
F(K, \nu, L)=\tilde{A} K^{\alpha}(\nu L)^{1-\alpha}=L c_{1}+L c_{2}+D+K \\
h=\eta(1-\nu) /\left(\xi S^{\gamma}\right) \\
S=(E / D)^{\chi} / \mu \\
E=z Y
\end{array}\right.
\end{array}
$$

The Lagrangian may be written as:

$$
\begin{array}{r}
\mathcal{L}=\phi\left(\log c_{1}+\theta \log c_{2}\right)+(1+\theta)(1-\phi)(\chi \gamma \log D-\chi \gamma \log F(K, \nu, L)+\log (1-\nu)) \\
+(1+\theta)(1-\phi) \log \frac{\eta \mu^{\gamma}}{\xi z^{\gamma \chi}}+\lambda\left(F(K, \nu, L)-L c_{1}-L c_{2}-D-K\right)
\end{array}
$$


First-order conditions are

$$
\begin{aligned}
& \phi c_{1}^{-1}=\lambda L \\
& \theta \phi c_{2}^{-1}=\lambda L
\end{aligned}
$$

that is

$$
\begin{aligned}
& c_{2}=\theta c_{1} \\
& \lambda\left(F_{K}^{\prime}(\cdot)-1\right)=(1+\theta) \chi \gamma(1-\phi) F_{K}^{\prime}(\cdot) / F(\cdot) \\
& (1-\phi)(1+\theta)\left(\gamma \chi F_{\nu}^{\prime}(\cdot) / F(\cdot)+(1-\nu)^{-1}\right)=\lambda F_{\nu}^{\prime}(\cdot) \\
& \lambda=(1+\theta) \chi \gamma(1-\phi) D^{-1}
\end{aligned}
$$

Equations (19) and (21) give:

$$
D=Y\left(1-1 / F_{K}^{\prime}(\cdot)\right)=Y-\alpha^{-1} K
$$

Furthermore, from (18), we obtain $\lambda=\frac{\phi}{c_{1} L}$, consequently

$$
D=\phi^{-1}(1+\theta) \chi \gamma(1-\phi) c_{1} L
$$

and consequently the market equilibrium gives

$$
Y=c_{1} L(1+\theta)\left[1+\phi^{-1} \chi \gamma(1-\phi)\right]+K
$$

that is

$$
c_{1} L=\frac{Y-K}{(1+\theta)\left[1+\phi^{-1} \chi \gamma(1-\phi)\right]}
$$

In the same way, the market equilibrium may be written as

$$
Y=(1+\theta) c_{1} L+K+Y-\alpha^{-1} K
$$

that is

$$
(1+\theta) c_{1} L=\left(\alpha^{-1}-1\right) K
$$

Consequently

$$
\frac{Y-K}{\left[1+\phi^{-1} \chi \gamma(1-\phi)\right]}=\left(\alpha^{-1}-1\right) K
$$


that is

$$
Y=\alpha^{-1} \phi^{-1}[(1-\alpha) \chi \gamma(1-\phi)+\phi] K
$$

Finally, equation (20) gives

$$
(1-\phi)(1+\theta)\left((1-\alpha) \gamma \chi+\frac{\nu}{1-\nu}\right)=\frac{\phi(1+\theta)}{\left(\alpha^{-1}-1\right)}(1-\alpha) Y / K
$$

that is

$$
\frac{\nu}{1-\nu}=\frac{\phi}{1-\phi} \quad \Rightarrow \quad \nu=\phi
$$

From (22), we can obtain the express of the steady-state physical capital in the centralized economy, denoted $K_{c}$ :

$$
\bar{K}=\left(\frac{A \alpha \phi}{(1-\alpha) \chi \gamma(1-\phi)+\phi}\right)^{1 /(1-\alpha)} \phi L
$$

and from $(22)$

$$
\bar{Y}=A^{1 /(1-\alpha)}\left(\frac{\alpha \phi}{(1-\alpha) \chi \gamma(1-\phi)+\phi}\right)^{\alpha /(1-\alpha)} \phi L
$$

Finally

$$
\bar{c}_{1}=\frac{(1-\alpha)}{\alpha(1+\theta)}\left(\frac{A \alpha \phi}{(1-\alpha) \chi \gamma(1-\phi)+\phi}\right)^{1 /(1-\alpha)} \phi
$$

and

$$
\bar{D}=\frac{(1-\alpha) \chi \gamma(1-\phi)}{(1-\alpha) \chi \gamma(1-\phi)+\phi} \bar{Y}
$$

\section{Subsidy to health-enhancing activities}

We re-write the competitive equilibrium assuming that the government subsidises the health-enhancing activities of agents by paying a subsidy $\tau^{\nu}$ to the opportunity cost of health-enhancing activities (that is the foregone wage $\left.\left(1-\nu_{t}\right) w_{t}\right)$ that is funded by a lump-sum tax denoted $a_{t}$.

The budget-constraint for the young agent born at period $t$ becomes:

$$
c_{1}+s_{t}+a_{t}=\nu_{t} w_{t}+\tau^{\nu}\left(1-\nu_{t}\right) w_{t}
$$

The maximization of lifetime utility gives $s_{t}=\frac{\theta}{1+\theta}\left(\nu_{t} w_{t}+\tau^{\nu}\left(1-\nu_{t}\right) w_{t}-a_{t}\right)$ and because government budget constraints require $a_{t}=\tau^{\nu}\left(1-\nu_{t}\right) w_{t}$, we obtain

$$
s_{t}=\frac{\theta}{1+\theta} \nu_{t} w_{t}
$$


and the individual labor supply is given by

$$
\nu_{t}=\frac{(1+\theta) \phi}{\theta(1-\phi)}\left(1-\tau^{\nu}\right) h_{t+1}
$$

In the steady-state equilibrium, the private health-status remains constant at:

$$
\begin{aligned}
h^{\star} & =\mathcal{H}(\tau) \equiv \eta\left[\left(1-\tau^{\nu}\right) \frac{\phi(1+\theta)}{(1-\phi) \theta}+\xi\left(\frac{(z / \tau)^{\chi}}{\mu}\right)^{\gamma}\right]^{-1} \\
\nu^{\star} & =\mathcal{V}(\tau) \equiv \phi\left[\phi+\frac{\xi(1-\phi) \theta}{\left(1-\tau^{\nu}\right)(1+\theta)}\left(\frac{(z / \tau)^{\chi}}{\mu}\right)^{\gamma}\right]^{-1}
\end{aligned}
$$

The higher the subsidy to health-enhancing activities, the higher the health-status in the steady-state and the lower the individual supply of labor $\nu^{\star}$.

The subsidy to health-enhancing activities that enables replication of the optimal allocation of time between health-enhancing activities and production (denoted $\overline{\tau^{\nu}}$ ) is such that $\nu^{\star}=\phi$, that is

$$
\overline{\tau^{\nu}}=1-\frac{\xi \theta}{(1+\theta)}\left(\frac{z^{\chi}}{\mu}\right)^{\gamma}\left(\frac{(1-\alpha) \chi \gamma(1-\phi)}{(1-\alpha) \chi \gamma(1-\phi)+\phi}\right)^{-\gamma \chi}
$$

\section{E The competitive equilibrium with leisure}

Up to this point, we investigated the competition for resources assuming that the time unit at the disposal of the agent is divided between two different "occupational" activities: production which enables an income to be earned and investment in health care which enables the agent to stay in good health.

In this section, we consider that each agent also values leisure-time and that she may adjust her leisure-time according to the level of her health-status: the healthier is the agent, the greater the utility of one minute of leisure.

To do so, we continue to denote $\nu \in] 0,1[$, the percentage of time the agent chooses in output production and we denote $u \in] 0,1[$ the percentage of time spent as investment in health status. Consequently $1-u-\nu \in] 0,1[$ represents the percentage of time

To keep things simple, preferences are written as follows

$$
\log \left(c_{1 t}^{\phi} h_{t}^{1-\phi}\left(1-u_{t}-\nu_{t}\right)^{\phi_{1}}\right)+\theta \log \left(c_{2 t+1}^{\phi} h_{t+1}^{1-\phi}\right)
$$

with $\phi_{1}>0$ captures the weight of leisure in utility.

The law of motion of the private health-status (equation 1) is modified as follows

$$
h_{t+1}-h_{t}=\eta u_{t}-\xi S_{t}^{\gamma} h_{t}
$$


and in the competitive equilibrium, the program of the agent is

$$
\begin{array}{r}
\max _{\left\{c_{1 t}, c_{2 t+1}, \nu_{t}, u_{t}\right\}} \log \left(c_{1 t}^{\phi} h_{t}^{1-\phi}\left(1-u_{t}-\nu_{t}\right)^{\phi_{1}}\right)+\theta \log \left(c_{2 t+1}^{\phi} h_{t+1}^{1-\phi}\right) \\
\text { s.t. }\left\{\begin{array}{l}
c_{1 t}+s_{t}=\nu_{t} w_{t} \\
c_{2 t+1}=\left(1+r_{t+1}\right) s_{t} \\
h_{t+1}=\eta u_{t}+\left(1-\xi S_{t}^{\gamma}\right) h_{t}
\end{array}\right.
\end{array}
$$

The expression of savings $s_{t}$ is the same than equation (3) and the percentage of time into output production $\nu_{t}$ is still given by equation (4). But now, the percentage of time in health care expenditure:

$$
u_{t}=1-\frac{\phi(1+\theta)}{\eta(1-\phi) \theta} h_{t+1}-\frac{\phi_{1}}{\eta(1-\phi) \theta} h_{t+1}=1-\frac{\phi(1+\theta)+\phi_{1}}{\eta(1-\phi) \theta} h_{t+1}
$$

and the percentage of time to leisure is

$$
1-u_{t}-\nu_{t}=\frac{\phi_{1}}{\eta(1-\phi) \theta} h_{t+1}
$$

The competitive equilibrium may be summarized by the following relations:

$$
\begin{aligned}
& K_{t+1}=(1-\alpha)\left(\frac{\theta}{1+\theta}\right)(1-\tau) A K_{t}^{\alpha}\left(\nu_{t} L\right)^{1-\alpha} \\
& \nu_{t}=\frac{\phi(1+\theta)}{\eta(1-\phi) \theta} h_{t+1} \\
& h_{t+1}=\eta u_{t}+\left(1-\xi S_{t}^{\gamma}\right) h_{t} \\
& u_{t}=1-\frac{\phi(1+\theta)+\phi_{1}}{\eta(1-\phi) \theta} h_{t+1} \\
& S_{t+1}=\left(\frac{z}{\tau}\right)^{\chi}+(1-\mu) S_{t}
\end{aligned}
$$

In the steady-state, $\nu, u, h, S, K, Y$ remain constant. Consequently, the stock of pollution in the steady-state $S^{\star}$ is always given by (9), and the private health-status is

$$
h^{\star}=\eta\left[\mathcal{B}^{\prime}+\xi\left(\frac{z^{\chi}}{\mu}\right)^{\gamma} \tau^{-\chi \gamma}\right]^{-1}
$$

with $\mathcal{B}^{\prime} \equiv \frac{\phi(1+\theta)+\phi_{1}}{(1-\phi) \theta}$. When leisure is taken into account, health care expenditure is lowered and the steady-state health status too, but the influence of the environmental tax is not modified.

The percentage of time allocated to the output production is:

$$
\nu^{\star}=\mathcal{B}\left[\mathcal{B}^{\prime}+\xi\left(\frac{z^{\chi}}{\mu}\right)^{\gamma} \tau^{-\chi \gamma}\right]^{-1}
$$


Finally, we obtain

$$
Y^{\star}=\mathcal{A}_{1}^{\prime}(1-\tau)^{\frac{\alpha}{1-\alpha}}\left(\mathcal{B}+\xi\left(\frac{z^{\chi}}{\mu}\right)^{\gamma} \tau^{-\chi \gamma}\right)^{-(1+\varepsilon)}
$$

with $\mathcal{A}_{1}^{\prime} \equiv \mathcal{B}^{\prime}\left(\frac{1+\theta}{\theta(1-\alpha)}\right) \mathcal{A}$.

Because $\mathcal{A}_{1}^{\prime}<\mathcal{A}_{1}$, the steady-state level of output is lower when leisure is taken into account, but the effect of the environmental taxation in the steady-state level of output and the expression of $\tau$, the environmental taxation under which a higher tax promotes economic activities, are not modified. 